\title{
Recollection and Familiarity in Aging Individuals with Mild Cognitive Impairment and Alzheimer's Disease: A Literature Review
}

\author{
Dorothee Schoemaker • Serge Gauthier • \\ Jens C. Pruessner
}

\begin{abstract}
Memory impairment is a central cognitive symptom in mild cognitive impairment (MCI) and Alzheimer Disease (AD). Recognition tasks are often used to characterize and define the nature of memory deficits. Dual-process theories posit that familiarity and recollection are independently involved in the recognition of previously encountered material and both contribute to successful recognition. Recent evidence indicates that there is a double dissociation in the neuronal substrates of those two processes. More precisely, it has been suggested that perirhinal and entorhinal areas are selectively involved in familiarity-based recognition, while the hippocampus is associated with recollection. Interestingly, these regions are among the first to be targeted by neurofibrillary tangles, one of AD's neuropathological hallmarks. Impairment in recognition performance can occur in the very early stages of $\mathrm{AD}$, such as MCI. To define the nature of recognition impairment in these clinical populations, we reviewed the current literature on familiarity and recollection performance in individuals with $\mathrm{MCI}$ and $\mathrm{AD}$. Together with clinical features, methodological factors are taken into consideration in the interpretation of findings.
\end{abstract}

Keywords Recognition · Memory · Alzheimer · Mild cognitive impairment · Recollection · Familiarity

D. Schoemaker $(\bowtie) \cdot$ S. Gauthier $\cdot$ J. C. Pruessner

McGill Centre for Studies in Aging, 6828 LaSalle Boulevard, Montreal H4H 1R3, Canada

e-mail: dorothee.schoemaker@mail.mcgill.ca

D. Schoemaker · J. C. Pruessner

Department of Psychology, McGill University, 1205 Dr. Penfield

Avenue, Montreal H3A 1B1, Canada

S. Gauthier · J. C. Pruessner

Department of Neurology and Neurosurgery, McGill University, 3801 University Street, H3A 2B4 Montreal, Canada

\section{Introduction}

Alzheimer disease (AD) is a progressive neurodegenerative condition that develops over years, if not decades. Before the onset of distinctive clinical manifestations, AD neuropathological processes are present for a prolonged period of time under a prodromal or pre-clinical stage (Jack et al. 2010; Bateman et al. 2012). The term "Mild Cognitive Impairment" (MCI) has been proposed to characterize individuals presenting objective cognitive deficits in the absence of impairment in daily functioning (Gauthier et al. 2006). MCI has gained considerable interest in the field of Alzheimer's research since, in comparison to cognitively intact elderly (CIE), this clinical population is at a much greater risk of progressing to AD (R. C. Petersen et al. 2001b; Wolf et al. 1998; Larrieu et al. 2002; R. C. Petersen et al. 2010). As a result, $\mathrm{MCI}$ is perceived by many to represent a transitional stage between healthy cognition and dementia (R. C. Petersen et al. 2001b; R. C. Petersen and Morris 2005; Morris 2006; Morris et al. 2001). The most frequent cognitive symptom that occurs in MCI is memory impairment (Ronald C Petersen et al. 1999). Furthermore, individuals with MCI who present memory deficits, also known as amnestic MCI (aMCI), have been found to be at a greater risk of conversion to $\mathrm{AD}$ than their non-amnestic counterparts (Sarazin et al. 2007; Ravaglia et al. 2006). Consequently, special consideration is now being given to memory in the evaluation of cognitive functioning in the aging population.

Performance on recognition tasks is often used to assess memory functions in normal (Grady et al. 1995) and clinical populations (Huron et al. 1995; Whittington et al. 2000; Mikhailova et al. 1996). According to dual-process theories, recognition of previously encountered material can be supported by two distinct processes: recollection and familiarity (Mandler 1980; Larry L. Jacoby 1991; Tulving 1985; Gardiner 1988). Recollection refers to recognition 
accompanied by a retrieval of contextual aspects associated with the encoding sequence. This type of recognition is often described as a vivid and conscious re-experience of a previous event. On the other hand, familiarity is recognition devoid of contextual information associated with the encoding episode. It corresponds to the feeling that a stimulus is familiar without being able to provide specific details related to the context or event where that stimulus was encountered. A number of dualprocess theories have been proposed to define the nature and contribution of both processes (see Yonelinas 2002 for a review). Most frequently, familiarity is conceived as a fast and automatic process whereas recollection is seen as a slower and more controlled process. Recollection is assumed to depend on a threshold process whereas familiarity is considered to follow signal-detection framework (Yonelinas et al. 1996). It is important to note that the dual-process theory of recognition is not endorsed unanimously by cognitive scientists. Some have argued that recognition is a continuous unidimensional process and that the variance observed in recognition judgments is associated with confidence or strength of the memory trace (Donaldson 1996; Dunn 2004; Slotnick 2009). Further, while acknowledging the implication of two different processes in recognition, other dual-process theories have proposed different models to characterize the interplay between familiarity and recollection. For example, the continuous dual-process model (Dede et al. 2013; Squire et al. 2007; Wixted 2007; Wixted and Mickes 2010) posits that recollection is a continuous, rather than dichotomous process. It also suggests that familiarity and recollection contribute simultaneously to recognition, rather than acting in an independent fashion. The existence of these various models and theories demonstrates that the exact nature of cognitive processes contributing to recognition is still a matter of debate. Nonetheless, a large body of evidence supports the involvement of two different processes in recognition, including neuropsychological (Yonelinas and Jacoby 1994, 1995; Yonelinas et al. 1998; McElree et al. 1999; King et al. 2004), lesion (Duarte et al. 2005; Yonelinas et al. 2002; Bowles et al. 2007; Bowles et al. 2010; Bowles et al. 2011; Martin et al. 2011; Aggleton et al. 2005; Vann et al. 2009), animal (Fortin et al. 2004; Sauvage et al. 2008; Easton et al. 2009), electrophysiological (Curran 2000; Curran and Cleary 2003; Curran et al. 2006; Speer and Curran 2007; Woodruff et al. 2006) and, more recently, neuroimaging studies (Skinner and Fernandes 2007; Yonelinas et al. 2005; Henson et al. 1999; Diana et al. 2007; Ranganath et al. 2004; Daselaar et al. 2006; Eldridge et al. 2000; Rauchs et al. 2007).

A number of studies indicate that the neural substrates of recollection and familiarity can be dissociated within medial temporal lobe regions (Skinner and Fernandes 2007; Yonelinas et al. 2005; Henson et al. 1999; Ranganath et al. 2004; Diana et al. 2007; Daselaar et al. 2006; Yonelinas et al. 2007). Neuroimaging studies have outlined an increased activation in hippocampal regions during the encoding of items which are later successfully recollected, whereas activation in perirhinal regions has been associated with items later recognized on the basis of familiarity (Ranganath et al. 2004; Daselaar et al. 2006; Eldridge et al. 2000). This finding was further corroborated in a meta-analysis by Diana and colleagues (Diana et al. 2007) revealing that, across studies, increased hippocampal and parahippocampal activation during encoding and recognition was consistently associated with recollection, but not with familiarity. On the other hand, perirhinal activation is frequently associated with familiarity, but rarely with recollection. Overall, results of functional imaging studies converge towards a functional doubledissociation between hippocampus and perirhinal areas, the former being associated with recollection and the latter with familiarity. This conclusion is further supported by human lesion studies that reveal an impaired familiarity judgment following perirhinal cortex lesions (Bowles et al. 2007; Bowles et al. 2010; Martin et al. 2011), whereas patients with lesions restricted to the hippocampus show recollection deficits with preserved familiarity (Bowles et al. 2010).

Interestingly, neuroanatomical regions that have been associated with familiarity and recollection are also among the very first to be affected by neurofibrillary tangles during the course of Alzheimer Disease. Indeed, as demonstrated by Braak \& Braak (1991), the perirhinal and entorhinal, regions that have been associated with familiarity-based recognition, are the first to show accumulation of neurofibrillary tangles. Accordingly, a study by Gomèz-Isla and colleagues revealed that individuals with a Clinical Dementia Rating scale score of 0.5 , which is indicative of MCI, had $32 \%$ less neurons in the entorhinal cortex than aged-matched healthy controls (GomezIsla et al. 1996). One could therefore expect to see impairments in familiarity in the very early stages of neurodegeneration (early MCI and perhaps even pre-MCI). Unfortunately, only a few studies have examined the impact of MCI and $\mathrm{AD}$ on performance in familiarity and recollection. Furthermore, results from these studies vary considerably, making it difficult to draw firm conclusions about the specific nature of recognition deficits in $\mathrm{MCI}$ and $\mathrm{AD}$ patients. Adding to this complexity, studies systematically investigating familiarity and recollection in individuals with MCI and AD have used inconsistent experimental paradigms to quantify familiarity and recollection processes. Due to these discrepancies, the nature of recognition impairments over the course of aging and dementia continue to remain ill-defined. The objective of this review is to provide a comprehensive summary of the current literature on the nature of recollection and familiarity performances in individuals with MCI and AD. We will examine whether both familiarity and recollection processes are impaired in MCI and AD and will highlight 
possible sources of variability in the results obtained by previous studies.

\section{Methods}

\section{Search Strategy and Inclusion Criteria}

Relevant articles published between January 1980 and May 2013 were identified with a search through online databases PUBMED and PsycINFO with the use of the following keywords: "familiarity," "recollection," "mild cognitive impairment," and "Alzheimer" using the Boolean 'AND' condition. Every article retrieved from this search was carefully reviewed. In order to avoid missing additional pertinent studies, the reference sections of the selected articles were also reviewed. Finally, to promote homogeneity between studies and to allow systematic comparison, articles were considered in this review only if they met the following criteria:

1. Articles had to be available in English.

2. Articles had to contribute previously unpublished findings (no review articles).

3. Articles had to provide quantitative estimates of familiarity and recollection.

4. Articles had to compare recollection and familiarity performances of healthy aging individuals and individuals with MCI and/or AD.

5. Articles had to make formal MCI and AD diagnosis, using established criteria.

6. Articles had to suspect the cause of MCI to be AD.

As a consequence of these criteria, articles including individuals with cognitive impairment due to other factors (e.g., hypoxia) were not considered. Also, studies making assumptions about the implication of familiarity or recollection on recognition performances, without any direct quantification of both processes, were disregarded. This was achieved via an internal review of the abstracts.

\section{Search Results}

Our search strategy using PUBMED and PsycINFO revealed 52 and 72 articles, respectively. Following the careful examination of each article however, only 14 studies met the inclusion criteria for this review. Population demographics and methodological details of these studies are summarized in Tables 1, 2, 3, 4 and 5. Due to the large amount of variation in the experimental paradigms and samples used to assess familiarity and recollection performances, as well as the clinical samples (age, MMSE, experimental design), we opted against computing and comparing effect sizes from each study.

\section{Study Samples Description}

Out of the 14 reviewed studies, five compared the recognition performances of cognitively intact elderly (CIE) to both MCI and $\mathrm{AD}$ patients; seven compared the recognition performances of CIE to MCI individuals, and two compared the recognition performances of CIE to AD patients. Demographic information of samples along with descriptive results of studies is presented in Table 1.

\section{Age}

There were important between-study variations in the average age of the experimental groups, which added a limitation to the comparability between studies, and the scope of this review. While the mean age of $\mathrm{AD}$ groups ranged from 71.9 to 80.3 years, the mean age of MCI groups ranged from 66.1 to 81.0 years and the mean age of CIE groups ranged from 66.67 to 81.0 years, across the 14 studies.

\section{Diagnostic Classification}

For the diagnosis of AD, all studies relied on the NINCDSADRDA criteria (McKhann et al. 1984). Two studies (Hudon et al. 2009; Winblad et al. 2004) also confirmed the diagnosis using DSM-IV criteria (American Psychiatric Association 1994). In studies examining performance in MCI, the most commonly used set of diagnostic criteria was the one proposed by Petersen (R. C. Petersen 2004; R. C. Petersen and Morris 2005; R. C. Petersen et al. 2001a). These criteria were used in nine out of the twelve studies (Hudon et al. 2009; Embree et al. 2012; Ally et al. 2009; Wolk et al. 2008; Wolk et al. 2013; Westerberg et al. 2006; Algarabel et al. 2012; Belleville et al. 2011; Serra et al. 2010). The Winblad (Winblad et al. 2004) and Lopez (Lopez et al. 2000) diagnostic recommendations were cited by three studies (Wolk et al. 2008; Anderson et al. 2008; Winblad et al. 2004). Across all studies, healthy elderly controls were selected based on performance on standard neuropsychological tests to document the absence of cognitive impairment.

\section{Subtype}

The majority of MCI groups were composed of both single and multiple-domain amnestic MCI (aMCI) (Hudon et al. 2009; Embree et al. 2012; Ally et al. 2009; Belleville et al. 2011; Wolk et al. 2008; Wolk et al. 2013). Even if not specified, MCI groups from the van der Meulen (2012) and Wolk (2011) studies most likely included both single and multiple-domain aMCI since impaired performance on cognitive functions other than memory were observed on neuropsychological tests results. Only three studies reported the proportion of single and multiple-domains included in their 


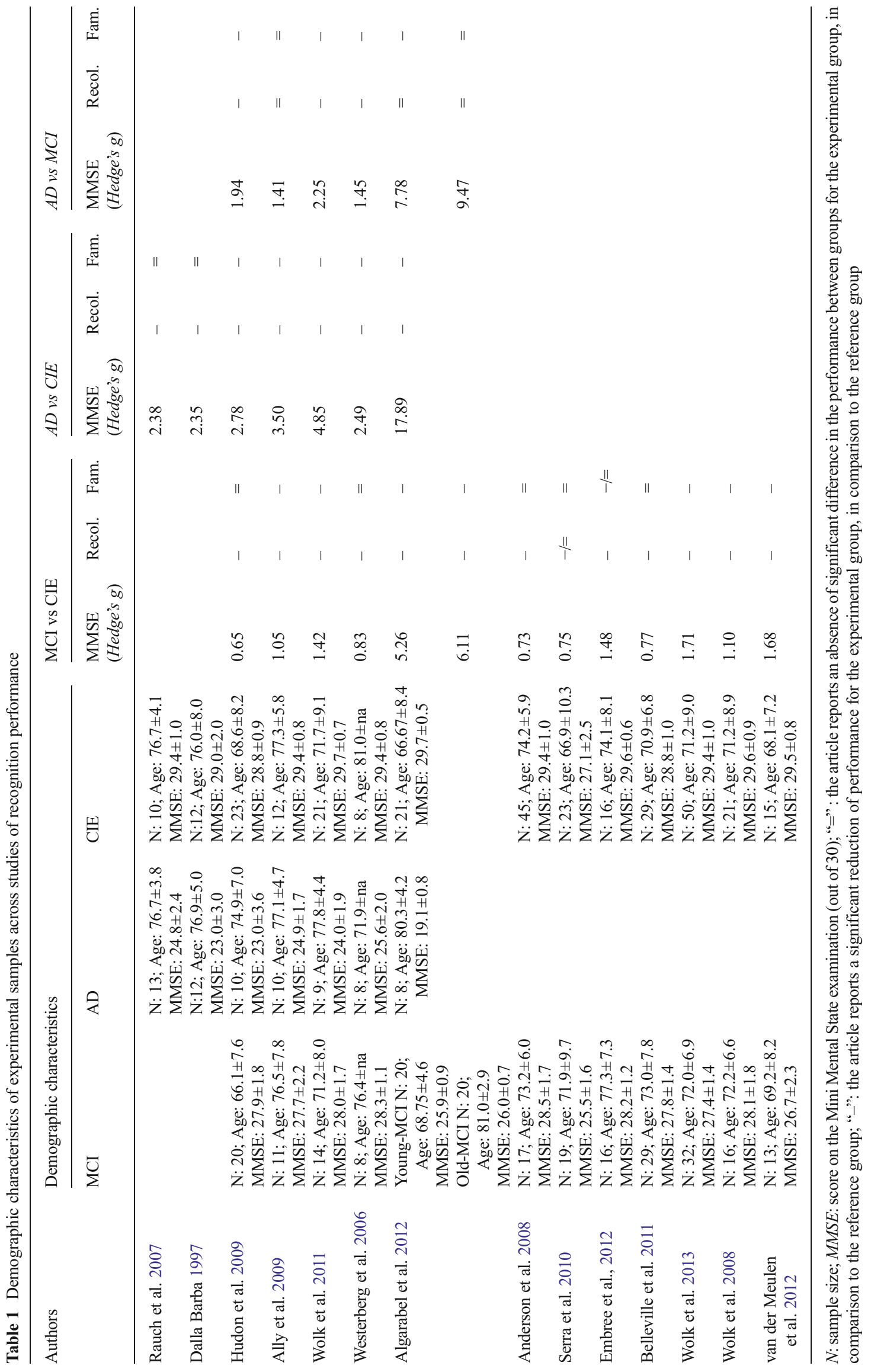


Table 2 Outcomes and methodological characteristics of studies using the Remember/Know paradigm

\begin{tabular}{|c|c|c|c|c|c|c|c|c|}
\hline \multirow[t]{2}{*}{ Authors } & \multicolumn{2}{|l|}{ Methodological characteristics } & \multicolumn{2}{|c|}{ MCI vs controls } & \multicolumn{2}{|c|}{$A D$ vs controls } & \multicolumn{2}{|c|}{$\mathrm{AD}$ vs $\mathrm{MCI}$} \\
\hline & Procedure & Stimuli & Recol. & Fam. & Recol. & Fam. & Recol. & Fam. \\
\hline \multirow[t]{4}{*}{ Dalla Barba 1997} & $\mathrm{RK}$ (free recognition) & Faces & & & - & $=$ & & \\
\hline & & Words & & & - & $=$ & & \\
\hline & RK (forced- choice recognition) & Faces & & & - & + & & \\
\hline & & Words & & & - & $=$ & & \\
\hline Rauchs et al. 2007 & RK & Words & & & - & $=$ & & \\
\hline Hudon et al. 2009 & RK & Words & - & $=$ & - & - & - & - \\
\hline \multirow[t]{4}{*}{ Belleville et al. 2011} & RK & Words & - & $=$ & & & & \\
\hline & & Pseudowords & - & $=$ & & & & \\
\hline & & Known melodies & - & $=$ & & & & \\
\hline & & Novel melodies & - & $=$ & & & & \\
\hline \multirow[t]{2}{*}{ Serra et al. 2010} & RK & Visual presentation -words to read & - & $=$ & & & & \\
\hline & & Visual presentation -anagrams & - & $=$ & & & & \\
\hline
\end{tabular}

$R K=$ remember/know paradigm; “=” : the article reports an absence of significant difference in the performance between groups; "-"”: the article reports a significant reduction of performance for the experimental group, in comparison to the reference group;; "+": the article reports a significant increase of performance for the experimental group, in comparison to the reference group

MCI samples (Hudon et al. 2009; Embree et al. 2012; Belleville et al. 2011). This information is relevant to the interpretation of results since individuals with multipledomain aMCI are likely to be at more advanced stages of cognitive impairment and, consequently, closer to dementia. The Serra (2010) and Anderson (2008) studies exclusively examined single-domain aMCI while Algarabel (2012) and Westerberg (2006) used multiple-domains MCI exclusively.

\section{Mini-Mental State Examination (MMSE)}

The MMSE is a brief screening tool that provides an overview of general cognitive functioning (Folstein et al. 1975). It is widely used in clinical and research settings. A score below 24 out of 30 is usually indicative of significant cognitive impairment or dementia (Mitchell 2009).

In the reviewed studies, the mean MMSE ranged from 18.6 to 25.6 for the $\mathrm{AD}$ groups, from 25.5 to 28.5 for the $\mathrm{MCI}$ groups, and from 27.1 to 29.7 for the CIE groups. As expected, there was considerably less variation in the average MMSE across CIE groups. The mean MMSE score of the CIE groups was higher than 29 in 12 out of the 14 reviewed studies. The mean MMSE of the CIE group in Serra et al. (2010) was noticeably low (27.1), indicating possible biases in the sample composition. The description of MMSE scores across studies highlighted 2 main findings: 1) there are important discrepancies in the general cognitive functioning of diagnostic groups across studies; 2) there is an overlap in average MMSE scores between the lowest functioning MCI

Table 3 Outcomes and methodological characteristics of studies using the Process Dissociation Procedure

\begin{tabular}{|c|c|c|c|c|c|c|c|c|}
\hline \multirow[t]{2}{*}{ Authors } & \multicolumn{2}{|l|}{ Methodological characteristics } & \multicolumn{2}{|c|}{ MCI vs controls } & \multicolumn{2}{|c|}{$A D$ vs controls } & \multicolumn{2}{|c|}{$\mathrm{AD}$ vs MCI } \\
\hline & Experimental paradigm & Stimuli & Recol. & Fam. & Recol. & Fam. & Recol. & Fam. \\
\hline \multirow[t]{2}{*}{ Serra et al. 2010} & \multirow[t]{2}{*}{ PDP } & Visual presentation -words to read & $=$ & $=$ & & & & \\
\hline & & Visual presentation -anagrams & $=$ & $=$ & & & & \\
\hline Anderson et al., 2008 & PDP & Words (visual and auditory presentation) & - & $=$ & & & & \\
\hline \multirow[t]{3}{*}{ Wolk et al. 2008} & PDP (associative) & Words & - & - & & & & \\
\hline & PDP (source vs. item memory) & Pictures & - & - & & & & \\
\hline & PDP (featural) & Words & $=$ & - & & & & \\
\hline Wolk et al. 2011 & PDP (associative) & Words & - & - & - & - & - & - \\
\hline Wolk et al., 2013 & PDP (associative) & Words & - & - & & & & \\
\hline
\end{tabular}

$P D P=$ Process Dissociation Procedure; "=” : the article reports an absence of significant difference in the performance between groups; “-": the article reports a significant reduction of performance for the experimental group, in comparison to the reference group 
Table 4 Outcomes and methodological characteristics of studies using a Receiver Operating Characteristic experiment

\begin{tabular}{|c|c|c|c|c|c|c|c|c|}
\hline \multirow[t]{2}{*}{ Authors } & \multicolumn{2}{|c|}{ Methodological characteristics } & \multicolumn{2}{|c|}{ MCI vs controls } & \multicolumn{2}{|c|}{$A D$ vs controls } & \multicolumn{2}{|c|}{$\mathrm{AD}$ vs $\mathrm{MCI}$} \\
\hline & Experimental paradigm & Stimuli & Recol. & Fam. & Recol. & Fam. & Recol. & Fam. \\
\hline Ally et al. 2009 & ROC & Words & - & - & - & - & $=$ & $=$ \\
\hline Embree et al. 2012 & ROC & $\begin{array}{l}\text { Words } \\
\text { Pictures }\end{array}$ & - & $\begin{array}{l}- \\
=\end{array}$ & & & & \\
\hline
\end{tabular}

ROC=Receiver Operating Characteristic; “=” : the article reports an absence of significant difference in the performance between groups; “-”: the article reports a significant reduction of performance for the experimental group, in comparison to the reference group

samples and the highest functioning AD samples. Similarly, there is an overlap in average MMSE score between the highest functioning MCI group and the lowest functioning CIE group.

To investigate the influence of MMSE score variations across groups and studies, between-group effect sizes were computed for each study using Hedge's g scores (Rosenthal 1991; Hedges et al. 1985). These effect sizes were calculated using the following formula:

Hedges $^{\prime} g=\frac{M t-M c}{\text { SDpooled }}$

where Mt is the mean MMSE of the patient group, Mc the mean MMSE of the control group, and SDpooled the weighted and pooled standard deviation. Therefore, these effect sizes represent the standardized mean difference in global cognitive functioning (as per the MMSE) between the different experimental groups in each study. The Hedge's g scores representing the difference between MMSE scores of control and AD groups varied between -2.35 and -17.89 . The comparison of MMSE scores between control and MCI groups yielded effect sizes ranging from -0.65 to -6.11 . Finally, effect sizes representing the difference between MMSE scores of MCI and AD groups varied between -1.41 and -9.47 . This important variation in the between-group effect sizes reconfirms the important discrepancies in the global cognitive functioning of the different experimental groups across studies and provides evidence that groups which are compared across studies are not equivalent.

\section{Methodological Considerations in the Assessment of Familiarity and Recollection}

Overview of Experimental Paradigms for the Assessment of Familiarity and Recollection

Over time, different experimental procedures have been developed in an attempt to quantify and distinguish the contribution of recollection and familiarity. Unfortunately, none of these procedures are assumed to convey pure and unbiased measures of recollection and familiarity and each of them with presents some limitations (for a review, see Yonelinas 2002). When reviewing selected articles, the most frequently used techniques to quantify familiarity and recollection can be classified into three different categories: the Rememberknow procedure (RK), the Process Dissociation Procedure (PDP) and Receiver Operating Characteristic experiments (see Tables 2, 3, 4 and 5).

The first commonly employed technique was the remember-know (RK) procedure developed by Tulving (1985). It was used by five of the 14 reviewed studies. The RK procedure requires participants to make a subjective judgment on the cognitive process that guides their recognition. More specifically, subjects are instructed to give a

Table 5 Outcomes and methodological characteristics of studies using other forms of experimental paradigms

\begin{tabular}{|c|c|c|c|c|c|c|c|c|}
\hline \multirow[t]{2}{*}{ Authors } & \multicolumn{2}{|c|}{ Methodological characteristics } & \multicolumn{2}{|c|}{ MCI vs controls } & \multicolumn{2}{|c|}{$A D$ vs controls } & \multicolumn{2}{|c|}{$\mathrm{AD}$ vs $\mathrm{MCI}$} \\
\hline & Experimental paradigm & Stimuli & Recol. & Fam. & Recol. & Fam. & Recol. & Fam. \\
\hline Westerberg et al. 2006 & Other & Pictures & - & $=$ & - & - & - & - \\
\hline Algarabel et al. 2012 & Other & Words & - & - & - & - & $=$ & $=$ \\
\hline van der Meulen et al. 2012 & Other & Pictures & - & - & & & & \\
\hline
\end{tabular}

"=" : the article reports an absence of significant difference in the performance between groups; "-": the article reports a significant reduction of performance for the experimental group, in comparison to the reference group 
"Remember" response when the item presented for recognition evokes conscious retrieval of some aspects of its encoding, and to give a "Know" response when the item is recognized but fails to elicit conscious recollection from the learning sequence. Since the instructions specify that an item should be called familiar only when not recollected, it has been suggested that the proportion of know responses are likely to provide an underestimation of familiarity (Yonelinas and Jacoby 1995). To overcome this bias, Yonelinas \& Jacoby (1995) proposed the "independence correction". In brief, this model assumes that familiarity is equivalent to the probability of giving a "Know" response in the absence of recollection. Hence, to control for the proportion of "Remember" response given by a participant, a formula is applied to the proportion of "Know" responses to derive "corrected" familiarity scores. A common criticism of the RK procedure is that it relies on participants' subjective assessment, and thus on the understanding of the instructions.

A second procedure commonly used to assess familiarity and recollection is the Process-dissociation procedure (PDP). It was used by five of the reviewed articles. This method was first proposed by Jacoby in 1991. In a typical PDP experiment, two sets of stimuli are presented under different encoding conditions. The recognition phase is also two-fold and includes an inclusion and exclusion condition. In the inclusion condition, participants are asked to endorse all previously studied items (i.e. targets) and to reject new items (i.e. distractor). In the exclusion condition, participants are asked to only endorse items from one of the two encoding conditions and to reject all the other items. It is assumed that, in the inclusion condition, both familiarity and recollection contribute to the recognition response. In contrast, in the exclusion condition, recollection is necessary to support an accurate judgment since the task instructions require access to contextual information about the encoding sequence. In comparison to the RK procedure, the PDP has been criticized to provide a too rigid and restricted definition of recollection.

The third procedure used to assess familiarity and recollection is based on the receiver operating characteristics (ROC) model. This technique was first used in favor of the dualprocess theory of recognition by Yonelinas in 1994. In typical ROC experiments, participants are asked to rate their recognition confidence on a Likert-type scale, usually ranging from "certain this item is new" to "certain this item is old". The hits and false alarms are then graphed as a function of response confidence and resulting curves are analyzed. The highthreshold model proposed by Yonelinas (1996) is frequently applied to derive familiarity and recollection estimates. One difficulty associated with the ROC model is that it requires a large number of recognition trials to yield a stable distribution over the range of confidence responses and to create valid curves for subsequent analysis. This possibly explains why ROC was used by only two of the reviewed studies (Embree et al. 2012; Ally et al. 2009). Furthermore, another limitation associated with ROC experiments is that they also include an element of subjectivity as they rely on patients' ratings of their own response confidence. However, confidence ratings are likely to be more intuitive to participants' than the more abstract differentiation between remember and know judgments.

Some of the reviewed articles employed other techniques to investigate familiarity and recollection. These have mainly relied on associative recognition or perceptual similarity to differentiate the contribution of familiarity and recollection processes. Methods from reviewed articles are presented in more detail in the next sections.

Summary of Studies Using the Remember-Know Procedure

The review of studies using the $\mathrm{R} / \mathrm{K}$ procedure (see Table 2 ) to assess the two recognition processes consistently supports a decline in recollection for $\mathrm{MCI}$ as well as AD patients. On the other hand, a preservation of familiarity in $\mathrm{MCI}$ and $\mathrm{AD}$ patients was revealed in all but one study.

Dalla Barba (1997) was among the very first to methodologically investigate recollection and familiarity in $\mathrm{AD}$ patients. He used two different versions of the RK paradigm to compare recollection and familiarity between $\mathrm{AD}$ patients and cognitively healthy elderly. Participants learned lists of 50 words and faces. The author first examined the RK response rates during a free recognition task, where participants had to correctly identify studied targets and reject lures presented one at a time. He also looked at the RK response rates during a forced-choice recognition task, where participants had to correctly differentiate a target stimulus from a lure when presented with pairs of stimuli. $\mathrm{R} / \mathrm{K}$ response rates for hits and false alarms were reported separately. Overall, in comparison to normal controls, the AD group showed a significant decrease in " $\mathrm{R}$ " response to targets. A preservation of " $\mathrm{K}$ " response was observed in all conditions except in the forced-choice recognition, where an increase in " $\mathrm{K}$ " response to targets was observed in $\mathrm{AD}$ patients in comparison to the control group. The author concluded that, when compared to controls, patients' recognition relied more on familiarity.

Rauchs et al. (2007) investigated familiarity and recollection in AD individuals in the context of a PET study. Participants learned a list of 15 words, which were repeated during five trials (based on the Grober and Buschke's procedure (Grober et al. 1988)). After a delay of $12 \mathrm{~h}$, participants were asked to differentiate the 15 studied words from 15 semantically related words, and 15 neutral distractors. In addition, they had to qualify their recognition strategy with either " $R$ " or " $K$ " responses and provide a justification for their R responses. Subjects were also given the opportunity to give a "Guess" response. Rates of " $\mathrm{R}$ " and " $\mathrm{K}$ " responses for target items were computed. No correction was made for false 
alarms. Overall, AD patients gave significantly fewer "R" responses. They also showed a tendency to provide more " $\mathrm{K}$ " responses than controls. Further, the researchers found that, while healthy controls used "R" responses more frequently than " $\mathrm{K}$ " responses, AD patients used " $\mathrm{R}$ " and " $\mathrm{K}$ " responses at a similar frequency. Finally, AD patients were found to be less efficient in justifying their " $\mathrm{R}$ " responses, which could indicate a lack of understanding of task instructions and a poorer capacity to accurately differentiate " $R$ " from " $K$ " recognition.

Hudon et al.[56] compared the performance of controls, $\mathrm{MCI}$ and $\mathrm{AD}$ patients on a RK task. During the encoding phase participants were instructed to memorize a set of 30 abstract and concrete words. At recognition, participants were presented with a list including all studied words and an equal number of lures and asked to only endorse previously studied words. For each endorsed word, participants had to give a "R" or " $K$ " response. Participants hit rates, sensitivity (A') and response bias (B"d) scores were calculated separately for "R" and " $\mathrm{K}$ " responses. " $\mathrm{K}$ " responses were also corrected according to the independence method (Yonelinas and Jacoby 1995), which takes into account the frequency of "R" responses to estimate familiarity. Results showed a significantly greater sensitivity for "R" response for CIE than for individuals with MCI or AD. Furthermore, sensitivity for " $\mathrm{R}$ " responses was significantly greater for MCI participants than for AD patients. Sensitivity for "K" responses did not differ between MCI and controls but was significantly lower for $\mathrm{AD}$ patients compared to both MCI and controls. When using the independence correction to estimate performance in familiarity, no group difference was found but a significant effect of age was observed, indicating that a greater age was associated with a decrease in familiarity. Because few other studies used the independence method to estimate familiarity and recollection, results based on sensitivity scores are presented in results tables of this article. Finally, taking into consideration that the $\mathrm{AD}$ group of this study was significantly older than the MCI group, it is hard to accurately interpret results yielded from this group comparison as any outlined differences could be explained by variation in age.

Belleville et al. (2011) compared performances in familiarity and recollection of MCI and healthy controls for verbal and musical material. Furthermore, they looked at the impact of material novelty on recognition performance. Overall, they observed a decline in recollection and preservation in familiarity in MCI compared to aged-matched cognitively intact individuals. The type of material did not affect any of the group differences. On the other hand, the novelty aspect of the studied material favored performances in recollection but did not seem to influence familiarity.

In one of their experiment, Serra et al. (2010) used the RK procedure to look at recollection and familiarity in individuals with MCI and cognitively intact elderly. During the encoding phase, participants had to learn words that were presented to them via auditory or visual presentation. Visual stimuli were either presented as words to read out loud, or as anagrams to be solved. During recognition, participants had to distinguish distractors from targets by giving a "R", "K", or new response. Further, when a word was identified as a target, participants were asked to define the context of encoding. $\mathrm{R}$ and $\mathrm{K}$ hit rates were computed separately for each presentation modality. Recollection and familiarity estimates were computed using the independence correction formula (Yonelinas and Jacoby 1995). For auditory stimuli, aMCI provided a greater proportion of " $\mathrm{K}$ " responses than controls. For visually presented words, MCI recollection was significantly reduced compared to controls. For both groups, recollection was greater for anagrams than read words. Familiarity estimates did not significantly differ between the control and the patient group. Finally, MCI patients were less likely than controls to correctly identify the encoding context of words classified as "R", however no group differences were reported for words classified as " $\mathrm{K}$ ".

Overall, all studies using the RK paradigm indicate a decline in recollection for $\mathrm{MCI}$ and $\mathrm{AD}$ patients and a preservation of familiarity in the MCI patients. For $\mathrm{AD}$ patients, two out of three studies suggest a preservation of familiarity-based recognition. The study by Hudon et al. (2009) is the only one demonstrating a decline in familiarity for $\mathrm{AD}$ patients compared to both cognitively intact controls and MCI patients. The RK paradigm seems less sensitive than other experimental paradigm to detect familiarity deficits in the course of $\mathrm{AD}$, as it is the only technique that suggests a preservation of familiarity in $\mathrm{AD}$ patients. It is also the only technique that systematically shows a preservation of familiarity in MCI across studies. However, the review of studies using the RK procedures showed that important methodological discrepancies exist across studies, which limits our ability to draw general conclusions from these results.

\section{Summary of Studies Using the Process-Dissociation Procedure}

Together with the RK procedure, the PDP procedure was found to be the most frequently used method to distinguish and quantify familiarity and recollection in $\mathrm{MCI}$ and $\mathrm{AD}$ patients. Overall, studies relying on PDP experiments yielded mixed findings (see Table 3) in the MCI population. Only one study assessed familiarity and recollection in AD patients using the PDP, making it difficult to draw conclusion related to this population. More details on each study follow.

Serra et al. (2010) compared recollection and familiarity estimates of MCI and CIE for auditorily presented words, visually presented words and anagrams. In the inclusion phase participants had to make an old/new judgment, whereas in the exclusion phase, participants had to endorse only auditorily 
presented words and reject the other ones. Recollection and familiarity estimates were computed according to the dualprocess signal detection theory (Jacoby et al. 1993). The results showed no significant group differences in familiarity for both stimulus types. While estimates of recollection did not differ for words, a tendency towards a decreased recollection for anagram words was outlined in MCI.

Anderson et al. (2008) also explored recollection and familiarity in a sample of MCI and CIE. The study procedure the authors employed was rather novel since it didn't include a separate encoding and recognition phase, rather the whole experiment comprised an inclusion and exclusion task. Participants studied words via auditory or visual presentation. Each word was presented twice over the learning sequence, either in the same or in a different presentation modality. In the inclusion phase, participants said "yes" when a word was repeated and "no" when it was presented for the first time, regardless of the presentation modality. In the exclusion phase, participants provided a "yes" answer only to words repeated in the same modality. Accordingly, they were asked to say "no" when a word was presented for the first time, or when a word was repeated in a different modality. Familiarity and recollection scores were computed for the exclusion condition alone as well as for both conditions combined. Overall, the pattern of findings was consistent; recollection was impaired in MCI while familiarity was preserved.

Wolk et al. (2008) used three variations of the PDP to explore recollection and familiarity performance in $\mathrm{MCI}$ and CIE individuals. In their associative PDP experiment, participants were presented with word-pairs and asked to form a mental image using both words. They were also instructed to make a judgment on the size of the items corresponding to the word. Word-pairs were presented either one or three times. In the recognition phase, participants had to differentiate new word-pairs, from rearranged or intact pairs. Familiarity and recollection estimates were computed in accordance with the dual-process signal detection model for the total, one-time repetition and three-time repetition conditions. Regardless of the encoding conditions, they observed a decrease in both familiarity and recollection in MCI compared to CIE. In their second experiment, they contrasted the memory for the item from the source memory. To do so, 80 colored pictures representing living and non-living items were presented four at a time, in each of the four quadrants of the screen. Participants were asked to name out loud the living items and to press buttons corresponding to their location on the screen. During the recognition phase, participants had to first discriminate between targets and distractors (i.e. old/new judgment). For each item identified as "old", participants were also asked to identify the screen quadrant in which the item was originally presented. The recollection and familiarity processes were estimated using the same equations as in the previous associative PDP paradigm. In their last experiment,
Wolk et al. (2008) developed a PDP experiment based on the features of the task's stimuli. During the encoding phase, participants studied 60 words printed either in green or red font. Half of the words were presented only once, while the other half were repeated four times over the encoding sequence. During the recognition phase, studied words as well as distractors were presented in black font. Participants were asked to only endorse words presented in one of the two colors. Performances of recollection and familiarity for words presented once, four times as well as the average performance were quantified. For all conditions, no significant group differences in recollection were found. In contrast, familiarity was significantly reduced in the MCI group across all conditions. Overall the results from all three PDP experiments performed by Wolk and colleagues suggest that familiaritybased recognition of MCI is as impaired, if not more, than recollection.

In a subsequent study, using the associative PDP as previously described, Wolk et al. (2011) investigated the neuroanatomical correlates of recollection and familiarity in $\mathrm{AD}, \mathrm{MCI}$ and healthy controls. Consistently with their previous report, the behavioral results of this study showed that, compared to CIE, both recollection and familiarity were impaired in the $\mathrm{AD}$ and $\mathrm{MCI}$ patients. Furthermore, in comparison to $\mathrm{MCI}$, the AD group showed a greater impairment in both recognition processes.

As part of a study examining the associations between recollection and familiarity and structural neuroimaging biomarkers of AD, Wolk et al. (2013) compared the recognition performance of controls and MCI individuals. Once again, they observed impairments in both recollection and familiarity in the MCI group.

In summary, studies relying on the PDP to assess familiarity and recollection have produced inconsistent results. It is possible that PDP is less sensitive to decline in recollection, as it is the only technique that reported preservation of familiarity in MCI patients. Wolk et al. $(2008,2011,2013)$ consistently outlined impairment in both recognition processes in MCI and AD patients. In contrast, using the PDP, Serra (2010) reported a preservation of both processes in the MCI group. Finally, Anderson (2008) outlined a decline in recollection and preservation of familiarity in their MCI sample. The variability in the methodological approach of these PDP experiments may have contributed to these discrepancies.

\section{Summary of Studies Using Receiver Operating Characteristics (ROC)}

Only two of the 14 studies relied on ROC experiments (see Table 4) to investigate familiarity and recollection in MCI and AD patients. Overall, these two studies outline a decline of recollection in both patients groups. The results of these studies also point toward familiarity impairment in MCI and 
AD. However, an experiment using pictures instead of words showed preservation in familiarity for MCI, indicating the type of stimulus used may influence familiarity judgments.

The first ROC study to investigate familiarity and recollection during pathological aging was conducted by Ally et al. (2009). Using ROC analyses, they compared the performance of controls, to MCI and AD patients. During the encoding phase, participants studied two 80-word lists under different encoding conditions. For the first list, they were asked to make a like or dislike judgment on presented words, a question that favors deep encoding. For the second list, they were asked to make a judgment on the number of syllables in each presented word, a question associated with a shallower form of encoding. During the recognition phase, participants were presented with a word-list that included all the studied words, and an equal number of new words. For each word, participants rated their recognition confidence on a scale from 1-6; ( 1 being "certain that the word is new" and 6 being "certain the word is old"). Familiarity and recollection estimates were computed for the shallow and deep encoding conditions separately, as well as for the average of both conditions. Both recollection and familiarity were found to be impaired in MCI and AD patients. However, no difference was found when comparing the two patient groups. In addition, in contrast to the control group, the MCI and AD patient groups did not seem to benefit from a deeper encoding.

Embree et al. (2012) also relied on the ROC technique to investigate familiarity and recollection in MCI and AD patients. For this study, participants were presented with 235 colored pictures or 235 words representing common objects. During the encoding phase, participants were shown each word for $2 \mathrm{~s}$, and each picture for $1 \mathrm{~s}$. For each stimulus, participants were asked to formulate a "like" versus "dislike" judgment. During the recognition phase the participants were presented a list of all the studied stimuli as well as the same number of distractors. For each stimulus, participants were asked to classify their answer on a scale from 1-6 (1 being "certain that the word is new" and 6 being "certain the word is old"). ROC curves were generated and analyzed separately for pictures and words. Results showed that recollection was impaired in the MCI patients, regardless of the stimulus type. Familiarity was also impaired in MCI for words, but not for pictures.

In summary, both studies that used ROC to investigate familiarity and recollection outlined a significant decline in recollection in MCI and AD patients, Regarding familiarity, Ally et al. (2009) study suggests a decline in the patient groups while Embree et al. (2012) report a selective impairment of familiarity for words. Overall, the low number of studies using the ROC procedure makes it difficult to draw conclusions about recollection and familiarity performances of MCI and $\mathrm{AD}$, when using ROC.

\section{Summary of Studies Using Other Methods}

Of the 14 reviewed studies, three studies used less common techniques to quantify the contribution of recollection and familiarity to recognition (See Table 5). These studies outline recollection impairment for $\mathrm{MCI}$ and $\mathrm{AD}$ patients, relative to the control group. All studies show familiarity impairments in AD patients, while 2 out 3 studies highlight familiarity deficits in MCI. A more thorough description of these studies is presented below.

Westerberg (2006) hypothesized that, when asked to discriminate between targets and visually similar distractors, an accurate yes-no recognition has to be mediated by recollection. In contrast, forced-choice recognition can be mediated by familiarity alone. Therefore, by comparing performance on those two tasks, recollection and familiarity can be assessed. To test this hypothesis, participants were presented with a set of 12 pictures representing either man-made or natural items. Each item was presented twice during the learning phase. Participants then completed a yes-no and a forced-choice recognition task. In the yes-no recognition task, participants were presented with a list of pictures including all studied targets and 36 visually similar distractors. Pictures were shown one at a time and participants responded "yes" if they believed that a picture had been previously seen and "no" if it was new. In the forced-choice recognition task, participants were presented with 4 highly similar pictures simultaneously, of which only one had been previously studied, and were asked to identify the target picture. Estimates of sensitivity (d') for the control, MCI and AD patients on the two recognition tasks were calculated and compared. The results showed impairments on the yes-no recognition task for the two patient groups, with an even greater impairment for $\mathrm{AD}$ patients relative to MCIs. In the forced-choice paradigm, impairment was noted for the AD group, while the MCI group was spared. Westerberg (2006) interpreted these results as a progressive decline in recollection in $\mathrm{MCI}$ and $\mathrm{AD}$, with preservation of familiarity-based recognition in $\mathrm{MCI}$ individuals.

Algarabel et al. (2012) contrasted performances on an associative and forced-choice recognition task to assess the contribution of familiarity in controls, young and old MCI individuals, and $\mathrm{AD}$ patients. Furthermore, they looked at the influence of two different experimental conditions. Participants first had to learn a list of word-pairs. In the associative-recognition task, they were asked to distinguish intact word-pairs from re-arranged ones. Since this requires participants to retrieve contextual information from the learning sequence, performance on this task was assumed to reflect recollection. In the forced-choice recognition task, word-pairs were presented and participants were asked to indicate which pair had been previously studied. However, this task was deceptive since half the word-pairs were never previously 
presented, but had a word with similar perceptual fluency as the ones presented during the study phase. This was meant to induce a feeling of familiarity in participants. In the associative recognition task, significant differences were found in the performance of controls and the three patient groups. No significant differences were found between patient groups since performance was poor overall. In the forced-choice recognition for studied words, the performance of the $\mathrm{AD}$ and MCI groups was lower compared to the control group. The forced-choice recognition for non-studied but perceptually similar words, revealed an inferior performance in both patient groups. These results are consistent with a prior study conducted by Algarabel in 2009. In brief, the authors found that $\mathrm{MCI}$ and $\mathrm{AD}$ patients were unable to benefit from perceptual familiarity to increase their recognition performance. In contrast, controls and non-amnestic MCI patients performed similarly, indicating a preserved familiarity. We did not include this study in the current review because it did not provide direct quantitative estimates of recollection and familiarity.

Lastly, van der Meulen et al. (2012) used an associative-memory paradigm to quantify recollection and familiarity. During the encoding condition, participants were asked to learn pairs of colored-pictures representing common objects or animals. The recognition phase was a forced-choice task whereby participants were presented with three pictures simultaneously. One served as a "cue" picture and participants were asked to determine which of the other two pictures was paired with the "cue" picture during the encoding phase. In other words, the triads always included a "cue", a target and a distractor and participants had to differentiate between the target and the distractor. The distractor was either a new picture or a picture presented previously together with another picture. This allowed for the differentiation of recognition based purely on perceptual familiarity from recollection based on encoding context. The results showed that participants' performance on trials with previously presented distractors was significantly lower in the MCI than in the control group, with the MCI patients showing impairments in recollection. These patients also showed performance impairments on trials with a new distractor, but to a lesser extent.

Overall, these studies relied on associative memory and perceptual similarity to estimate the contribution of recollection and familiarity. Using these techniques, all three studies observed a decline in recollection. Two studies point towards a decline in familiarity in familiarity for MCI individuals, while Westerberg (2006) study demonstrated preservation. However, due to the important methodological differences across those studies it is hard to draw comparisons and assess the factors influencing their results.
The Effect of Stimulus Modality on Familiarity and Recollection Performance

Across studies, words were the most commonly used stimuli, followed by pictures of common objects. Only a few studies have looked at the influence of stimulus modality on recollection and familiarity performance (Embree et al. 2012; Dalla Barba 1997; Belleville et al. 2011). To investigate the effect of novelty on recollection and familiarity, Belleville et al. (2011) used words, pseudowords, known melodies and novel melodies. Across experimental groups, they found that the novelty of the material favored recollection but not familiarity. They also revealed a main effect of material, indicating a better recognition for verbal than musical stimuli. Embree et al. (2012) looked at recollection and familiarity performance separately for words and pictures. Across experimental groups, they found that familiarity was higher for pictures than words. They also highlighted a group-bymaterial interaction indicating impairment in familiarity for words, but not for pictures in MCI individuals. Recollection performance was not affected by stimulus modality. Dalla Barba (1997) looked at familiarity and recollection for words and unfamiliar faces. Overall, a similar pattern of findings was highlighted for both words and faces. However, in the forcedchoice recognition experiment, AD patients gave significantly more " $\mathrm{K}$ " responses than controls to target faces, while no significant differences were found for words. Overall, these findings suggest that the type of stimuli used to assess familiarity and recollection might influence the results.

\section{The Effect of MCI and AD on Familiarity and Recollection Performance}

In this section, we further describe differences in familiarity and recollection between groups. Globally, studies highlight a decline in recollection for AD and MCI patients.

All studies comparing AD patients to controls have reached the conclusion that recollection is impaired in AD patients (Rauchs et al. 2007; Hudon et al. 2009; Ally et al. 2009; Westerberg et al. 2006; Algarabel et al. 2012; Wolk et al. 2011; Dalla Barba 1997). As for performance on familiarity tasks, 5 out of 7 studies demonstrated a decline. Rauchs et al. (2007) failed to find significant differences in familiarity between AD patients and CIE. They even found a tendency towards an increased familiarity in AD patients, but this did not remain significant after Bonferoni correction was applied to correct for multiple comparisons. Similarly, Dalla Barba (1997) concluded that while AD patients' recollection was impaired across experimental conditions, familiarity-based recognition was globally preserved. An increased proportion of " $\mathrm{K}$ " responses for $\mathrm{AD}$ patients 
was also observed in the forced-choice recognition for faces. These two studies discuss the counter-intuitive findings as a compensatory mechanism used by $\mathrm{AD}$ patients: to compensate for recollection deficits, these patients rely more on familiarity to support their recognition. However, both of the latter studies did not correct for false alarm rate, which is known to be increased in AD patients (Budson et al. 2000). Finally, Hudon et al. (2009) found a decrease in familiarity when using the sensitivity index (A'). However, in a later analysis of their results using the independence correction, which corrects for an underestimation of familiarity associated with task instructions, the group difference was no longer found to be significant.

In contrast, studies comparing recognition performances of MCI and CIE patients produced more variable results. Of the two studies examining single-domain aMCI, neither found significant impairment in familiarity compared to healthy aged matched controls (Serra et al. 2010; Anderson et al. 2008). However, it is important to note that for both of these studies, the standardized difference in the mean MMSE scores between the MCI and CIE group was not very high (effect size lower than 0.75 ). With respect to performances in recollection, Anderson et al. (2008) reported declining performance in the MCI group, while Serra's (2010) results point towards a preservation of recollection when using the PDP, and a decline when using the RK procedure. Of the reviewed articles, the latter is the only one that proposes a preservation of recollection in MCI. However, as mentioned previously, the mean MMSE score of the control group in Serra et al. (2010) is rather low, thereby suggesting that some of their elderly controls might have presented with cognitive impairments. Of the studies using samples of both multiple and single domain MCI, five concluded that familiarity was significantly impaired in MCI patients when compared to CIE patients (Ally et al. 2009; Wolk et al. 2013; Wolk et al. 2008; Wolk et al. 2011; van der Meulen et al. 2012) while two found a preservation of familiarity in the MCIs (Hudon et al. 2009; Belleville et al. 2011). However, in these studies the discrepancy between MMSE scores of patients and controls was not as large as other studies (effect size lower than 0.8).

The results obtained by Embree et al. (2012) vary according to the modality of stimuli used in the experimental paradigm. More specifically, the researchers found a preservation of familiarity in pictures and impairment for words, compared to the control group. In the two studies including multipledomains aMCI individuals, Algarabel et al. (2012) found deficits in both recollection and familiarity, whereas Westberger (2006) found deficits in recollection with preservation of familiarity. However, in the Westberger (2006) study, although not statistically significant, the control group was noticeably older than the MCI groups (81.0 versus 76.4 years). Because recognition performance has been shown to be affected by age (Rabinowitz 1984), this could have contributed to the absence of differences in familiarity performance between groups.

Results of studies comparing recognition performance of MCI and AD patients have produced highly variable results. Looking at recollection, two out of five studies do not indicate deterioration in $\mathrm{AD}$ patients' recollection relative to MCIs (Ally et al. 2009; Algarabel et al. 2012). In contrast, the three remaining studies suggest a progressive decline in recollection from MCI to AD (Hudon et al. 2009; Westerberg et al. 2006; Wolk et al. 2011). Observed differences in the global cognitive functioning between $\mathrm{AD}$ and $\mathrm{MCI}$ groups do not seem to explain variations in these results. For familiarity, three of the five studies show additional impairments in AD patients relative to MCI (Hudon et al. 2009; Westerberg et al. 2006; Wolk et al. 2011). In contrast, Ally et al. (2009) failed to find significant differences in familiarity between those two groups. Finally, Algarabel et al. (2012) outlined significant differences in familiarity between groups only when AD patients were compared with younger MCI patients, thereby indicating that age itself may play an important factor in familiarity deficits.

Overall, the set of diagnostic criteria used for classification of patients does not seem to influence the pattern of findings related to recollection and familiarity performances. All studies show impairments in recollection in $\mathrm{MCI}$ and $\mathrm{AD}$ patients relative to CIE. However, results obtained by Serra et al. (2010) suggest a preservation of recollection for MCI individuals when assessed with the PDP procedure. Results associated with performance in familiarity tasks are more variable. For $\mathrm{AD}$ patients, most studies point towards a decline in familiarity. However, two studies suggest preservation, or even an increase in familiarity-based recognition (Rauchs et al. 2007; Dalla Barba 1997). For studies investigating familiarity performances in MCI, five observed a preservation of familiarity (Hudon et al. 2009; Belleville et al. 2011; Westerberg et al. 2006; Anderson et al. 2008; Serra et al. 2010) and six reported a decline (Ally et al. 2009; Wolk et al. 2011; Wolk et al. 2013; Wolk et al. 2008; van der Meulen et al. 2012; Algarabel et al. 2012). The four studies with a MMSE effect size lower than 0.8 did not find any differences in familiarity performance between control and patient groups (Hudon et al. 2009; Belleville et al. 2011; Anderson et al. 2008; Serra et al. 2010). Thus, an increased discrepancy in cognitive functioning between control and patient groups (MCI or AD) seems to be associated with findings of familiarity deficits. Finally, when investigating differences in recollection and familiarity between $\mathrm{AD}$ and MCI, studies have yielded very inconsistent results, indicating the need for more research.

\section{Discussion}

The objective of this review was to provide an overview of the current literature on familiarity and recollection in individuals 
with MCI or AD. To our knowledge, this is the first article that reviews this particular set of studies.

Our review suggests that, in AD patients, both recollection and familiarity are affected. Indeed, studies have systematically outlined a decline in recollection in this population. The majority of reviewed studies investigating AD patients have also indicated a decline in familiarity-based recognition. This review also provides strong evidence for recollection impairments in individuals with MCI, with all studies showing a decline in recollection for MCI individuals relative to agematched controls. With regard to familiarity in individuals with MCI, results are more inconsistent: six studies demonstrated a decline in familiarity-based recognition in MCI individuals (Ally et al. 2009; Wolk et al. 2011; Wolk et al. 2013; Wolk et al. 2008; van der Meulen et al. 2012; Algarabel et al. 2012), whereas five studies point towards preservation (Hudon et al. 2009; Westerberg et al. 2006; Anderson et al. 2008; Serra et al. 2010; Belleville et al. 2011). Finally, Embree et al. (2012) found that MCIs' familiarity-based recognition varies according to the modality of the stimulus used. Interestingly, while no quantitative estimation was included in this review, it seems that a greater difference in global cognitive functioning between control and MCI groups is associated with an increased likelihood of detecting significant impairments in both familiarity and recollection will be observed. This suggests that MCI individuals at a more advanced stage of $\mathrm{AD}$ are more likely to present significant familiarity deficits relative to age-matched controls.

The results of this review demonstrate that familiarity and recollection are differentially affected by progressing $\mathrm{AD}$ neuropathology. While recollection is broadly affected through all stages of the disease process, familiarity deficits seem to be present only at more advance stages of cognitive impairment. This supports the involvement of distinct processes in recognition and is in agreement with dual-process theories. Past researches have highlighted a possible dissociation in the neural substrates of familiarity and recollection. More precisely, the available evidence suggests that the hippocampus region supports recollection (Bowles et al. 2010; Bowles et al. 2011). On the other hand, familiarity has been associated with perirhinal/entorhinal areas (Bowles et al. 2007; Martin et al. 2011). Interestingly, these regions are affected in the early stages of AD. Due to an earlier and more pronounced neuropathological alteration of perirhinal/ entorhinal areas relative to the hippocampus in the course of $\mathrm{AD}$, we originally hypothesized that familiarity deficits would be a prominent features in individuals with MCI. However, the review of studies revealed mixed findings and does not always support the presence of familiarity deficits in MCI individuals. To explain this pattern of deficits, we propose a model that can be compared to the progression of neurological changes that occur as a function of healthy or pathological aging (Figs. 1a and b). While perirhinal/entorhinal areas are selectively affected by AD, hippocampal structural and functional impairments are known to occur in normal aging (Driscoll et al. 2003; Scahill et al. 2003; Raz et al. 2005). Due to a decreased efficiency in hippocampal functioning, it is expected to find recollection impairment in normal aging individuals. Consistently, previous studies looking at the effect of normal aging on recollection have outlined a decline in recollection-based recognition in this population (McCabe et al. 2009; Davidson and Glisky 2002; Anderson et al. 2008; Prull et al. 2006). Importantly, it has been suggested that, in comparison to young individuals, healthy aging individuals rely more on familiarity in recognition tasks (Bastin and Van der Linden 2003; Sander M Daselaar et al. 2006; Jennings and Jacoby 1997). This increased reliance on familiarity might constitute a compensatory mechanism employed by healthy aging individuals to preserve their recognition performance, despite a decreased recollection. In accordance with this idea, many studies have found a preserved recognition performance in cognitively intact aging individuals (Schonfield and Robertson 1966; Fabiani and Friedman 1996; Craik and McDowd 1987). This amplified use of familiarity can also be associated with the increased rate of false alarms outlined by many studies investigating recognition memory in aging individuals (Schacter et al. 1997; McCabe et al. 2009). While this line of evidence points toward an increased reliance on familiarity during normal aging, this has not been studied comprehensively. Neural mechanisms by which familiarity becomes more potent during aging also remain unidentified. Different pathways could contribute to this compensatory strategy. First, it is possible that in the course of normal aging, due to a decreased efficiency in hippocampal functioning, there is a greater involvement of entorhinal/perirhinal areas during encoding and subsequent recognition leading to more prominent familiarity judgments. However, a greater reliance on familiarity could also be the consequence of a global weakening of neural traces associated with contextual information (due to impairment in hippocampus functioning) contributing to recollection judgments, resulting in more familiarity-like judgments. To summarize, our model proposes that while normal aging is associated with impairment in recollection memory, recognition performance remains unaffected due to an increased reliance on familiarity to support recognition (Fig. 1a).

In the course of $\mathrm{AD}$, both perirhinal/entorhinal and hippocampal regions become affected by tauopathies at a very early stage (Braak and Braak 1991). Further, the more advanced the disease, the more severe will be the pathological alteration in these regions. Thus, in addition to age-related changes, individuals with progressing $\mathrm{AD}$ also present neuropathological alterations in the hippocampus (Fig. 1b). Hence, with regards to recollection performance, it is expected to find more severe deficits in these individuals, when compared to cognitively intact individuals. Consistently, studies reviewed in this article 
Fig. 1 Theoretical model to explain the involvement of recollection and familiarity to recognition performance in the continuum from normal aging to Alzheimer Disease. Functional neuroanatomical studies suggest that entorhinal/perirhinal areas and hippocampus differentially contribute to familiarity and recollection. Consequently, the changes in recognition performance are presented in conjunction with the progression of neuropathological impairments in $\mathrm{AD}$ in those medial temporal lobe regions. Ranging from young adulthood to normal aging (a), or to Alzheimer Disease (b), the differential contribution of familiarity and recollection to the overall recognition performance is displayed in conjunction with progression of volume loss in the hippocampus and entorhinal/ perirhinal areas
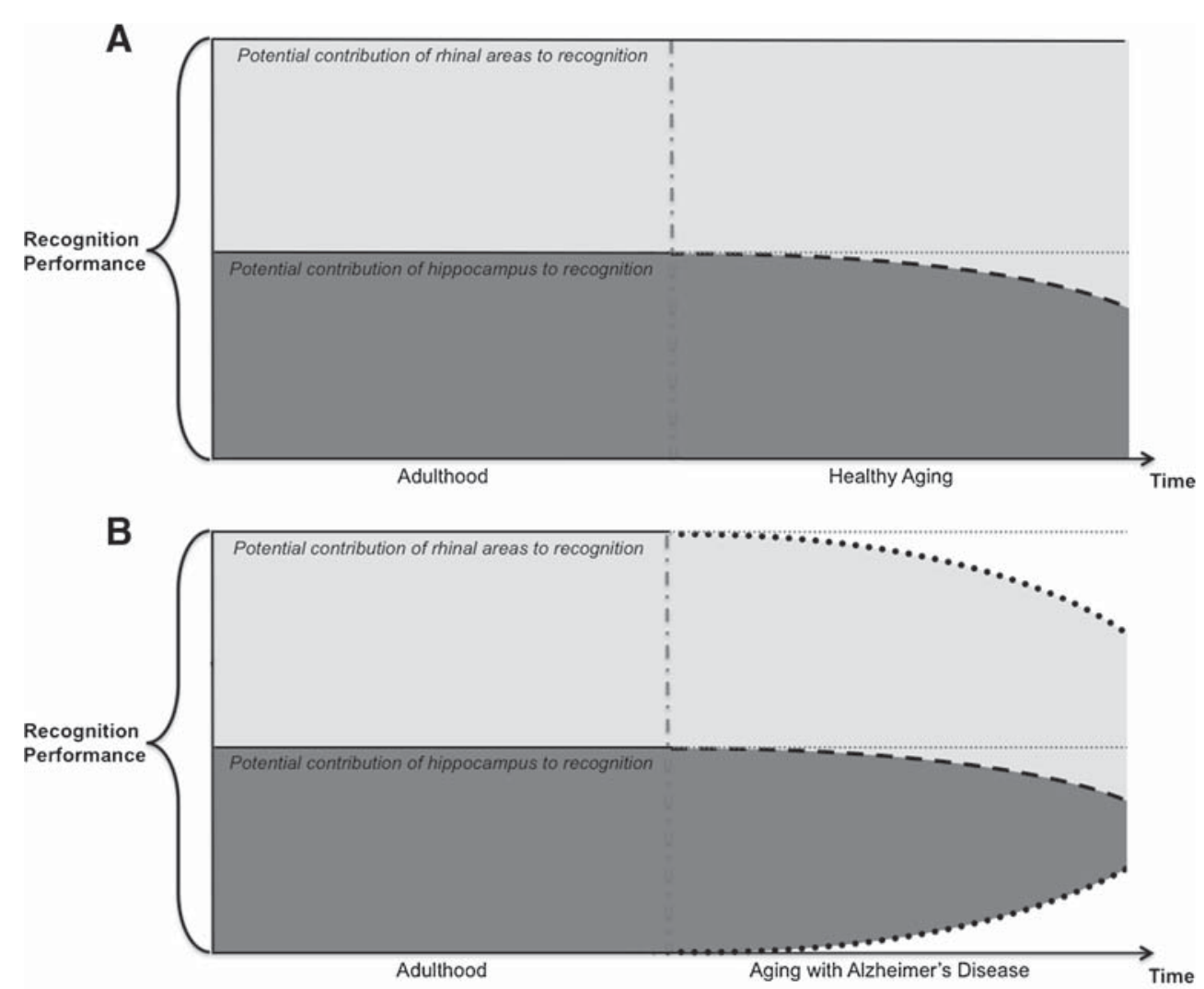

LEGEND

Contribution of familiarity to recognition performance

Contribution of recollection to recognition performance

- - Consequences of neural changes occurring during normal aging

.... Consequences of neuropathological changes occurring during Alzheimer Disease have shown that individuals with $\mathrm{MCI}$ and $\mathrm{AD}$ present impairments in recollection-based recognition. In addition, AD is also associated with neuropathological alteration of rhinal areas. Consequently, it would be also expected to find familiarity impairments during AD. In line with this, our literature review suggests that patient groups with lower cognitive functioning (as per the MMSE), in comparison to CIE, are more likely to present familiarity deficits. In clinical AD, where overt cognitive and functional deficits can be observed, both the rhinal areas and the hippocampus are severely affected by pathological changes and neither recollection nor familiarity can sufficiently support the recognition of previously encountered material (Fig. 1b). In accordance with that idea, recognition impairments have previously been found in individuals with AD (R. C. Petersen et al. 2010; Collie and Maruff 2000; Greenaway et al. 2006).

To summarize, according to this hypothetical model, recollection is affected during normal aging due to age-related hippocampal structural and functional changes, while there is an over-reliance on familiarity to support accurate recognition judgments. During progression of $\mathrm{AD}$, recollection is affected by both age-related and neuropathological changes in the hipocampal region. Familiarity is selectively affected by the presence of $\mathrm{AD}$ neuropathology: as the disease progresses, more pronounced deficits in familiarity can be observed.

Taken together, this theoretical model aims to explain the pattern of recollection and familiarity deficits outlined by this literature review. While we acknowledge that the reviewed studies do not provide enough evidence to support the accuracy of this model, we hope that it can contribute to guide future research looking at the effects of $\mathrm{AD}$ on recollection and familiarity.

Other factors and methodological considerations might have influenced the findings of this review and contributed to the highlighted pattern of familiarity and recollection deficits in individuals with MCI and AD.

First, results concerning recognition performance in $\mathrm{MCI}$ were found to be more variable. MCI population is known to be highly heterogenous. Accordingly, a follow-up study on a MCI cohort revealed that $15 \%$ developed a different form of dementia and $41 \%$ remained stable over a time frame of 4-6 years (Hansson et al. 2006). Similarly, studies have found that an important percentage of MCI (30-60 \%) individuals do not arbour AD neuropathological features, such as amyloid 
deposition (Chételat et al. 2013). Those results highlight that, in a considerable proportion of MCI individuals, cognitive impairments are associated to other factors than pre-clinical AD. Consequently, MCI samples from the reviewed studies might have included, at different extent, individuals with cognitive impairments not associated with pre-clinical AD. It is possible that familiarity deficits are specific to MCI due to pre-clinical $\mathrm{AD}$ and insensitive to other types of MCI. To maximize the interpretability of findings related to the effect of $\mathrm{AD}$ on these two recognition processes, follow-up studies or studies including only MCI with approved AD biomarkers (such as FDG/ ${ }^{11} \mathrm{C}$-PiB PET or CSF T-tau, P-tau and A $\beta 42$; Albert et al. 2011) should be performed.

Second, there were important between-studies differences with regard to demographic features of experimental groups. The most salient discrepancy was in the global cognitive state of the different patient groups. The average MMSE of patients and control groups varied widely across studies. With regards to individuals with MCI this is particularly relevant, as it is possible that MCI samples with higher cognitive functioning, relative to the control group, include a higher proportion of non-converter MCI (Nordlund et al. 2010). Consistently, individuals with more advanced cognitive impairment are likely to present more severe and widespread AD neuropathological alterations (Seo et al. 2007; Csernansky et al. 2004; Landau et al. 2012), indicating a greater risk of conversion to dementia. Furthermore, MCI groups were composed of different subtypes; while some studies only used single-domain amnestic MCI, others used only multiple-domain MCI or both. Those different clinical populations have been associated with different rates of conversion to $\mathrm{AD}$ (Fischer et al. 2007). Consequently, the presence of different subtypes of MCI across studies suggests that experimental groups varied in term of neuropathological severity or rates of non-AD related MCI. Finally, important between-studies variations in the age of experimental groups were also noted. As demonstrated by previous studies, age seems to influence recollection and familiarity processes (Bastin and Van der Linden 2003; Daselaar et al. 2006; Jennings and Jacoby 1997). Accordingly, age itself can influence findings from the different studies.

Thirdly, the presence of important methodological discrepancies makes it extremely challenging to systematically compare results across studies. The most common experimental paradigms used to investigate familiarity and recollection performance were the RK task, and the PDP. However, tasks based on confidence ratings (ROC) and on associative memory were also employed. Our review showed that there was a possible effect of the testing paradigm. While all studies using the RK showed a preservation of familiarity for MCI in comparison to CIE, studies relying on other methodological paradigms yielded more variable results. Similarly, studies using the RK task were the only ones to outline a preservation of familiarity in $\mathrm{AD}$ patients. Consequently, it is possible that this technique is less sensitive to detect impairments in familiarity in individuals affected with cognitive impairments. Indeed, for $\mathrm{MCI}$ and $\mathrm{AD}$ patients, RK task instructions might be too complex to understand. Accordingly, Rauchs et al. (2007) outlined a decreased capacity of patients to correctly justify their answer and differentiate " $\mathrm{R}$ " from " $\mathrm{K}$ " responses. On the other hand, studies using the PDP were the only ones to highlight preservation in recollection for individuals with MCI. This technique is therefore less sensitive to detect impairment in recollection in this patient population. Overall, this suggests that the choice of the experimental paradigm might affect the global assessment of familiarity and recollection. Not every paradigm might be equally suitable to detect differences in these patient populations. This could also reflect the fact that these paradigms measure different elements of recognition. Furthermore, even within similar experimental paradigms, a number of differences were found in terms of encoding of recognition conditions, the time interval between encoding and recognition, the time of stimulus presentation, and the type of stimulus used. All these factors could have contributed to the variability in findings across studies.

Another limitation associated with this review lies in the quantification of familiarity and recollection estimates. While some authors based their familiarity and recollection estimates on hit and false alarms rates, others used more complex formulas, e.g., the independence correction, to quantify the contribution of each process. Consequently, it becomes difficult to systematically compare these quantifications across studies.

For all of these previous methodological discrepancies, we did not quantitatively compare results across studies, as it might have provided biased estimates of group differences in familiarity and recollection. This does limit the scope of this review. Finally, another limitation inherent to this review is that it was performed solely in light of the high threshold dualprocess theory of recognition, which perceives familiarity and recollection as being intrinsically independent and dissociable. Other models have been proposed to characterize recognition memory and some studies have suggested that the high threshold dual-process theory of recognition might provide a biased theoretical framework (Dede et al. 2013; Squire et al. 2007; Wixted 2007; Wixted and Mickes 2010).

In the hope of finding cognitive symptoms that can help predict conversion to dementia, many studies have characterized cognitive performance of individuals with MCI and AD. While recollection has consistently been found to be impaired in these two clinical populations, our review suggests that familiarity deficits arise with more severe cognitive impairment. Hence, it could be a useful clinical marker of individuals who are closer to convert to dementia. However, more longitudinal studies need to be performed to clarify the nature of 
familiarity performance in the course of $\mathrm{AD}$. The relatively small number of studies that have investigated familiarity and recollection in individuals with $\mathrm{MCI}$ and $\mathrm{AD}$ makes it difficult to define the exact nature of recognition deficits in those populations. In addition, the reviewed studies have methodological shortcomings and confounding variables that have to be considered before general conclusions can be drawn. Replication studies and further research comparing different experimental paradigms (such as "RK", "PDP" and "ROC"), clinical populations (MCI with confirmed AD neuropathology versus non- $\mathrm{AD}$ related $\mathrm{MCI}$ ), procedures (such as the type of stimuli and the encoding and recognition instructions), and theoretical models are needed before broader use of familiarity and recollection assessments in clinical settings can be envisioned.

Acknowledgments We would like to thank Nida Ali for her inputs and comments on earlier versions of the manuscript.

Funding Information DS was supported by scholarships provided by the Fonds de recherche du Québec-Santé (FRQS) and the Canadian Institutes of Health Research (CIHR). JS holds a "Chercheur National" from the Fonds de recherche du Québec-Santé (FRQS). This work was supported in part by the Le Fonds de recherche du Québec-Santé (FRQS) grant 23638 .

\section{References}

Aggleton, J. P., Vann, S. D., Denby, C., Dix, S., Mayes, A. R., Roberts, N., et al. (2005). Sparing of the familiarity component of recognition memory in a patient with hippocampal pathology. Neuropsychologia, 43(12), 1810-1823.

Albert, M. S., DeKosky, S. T., Dickson, D., Dubois, B., Feldman, H. H., Fox, N. C., et al. (2011). The diagnosis of mild cognitive impairment due to Alzheimer's disease: recommendations from the national institute on aging-Alzheimer's association workgroups on diagnostic guidelines for Alzheimer's disease. Alzheimer's \& Dementia, 7(3), 270-279.

Algarabel, S., Escudero, J., Mazón, J. F., Pitarque, A., Fuentes, M., Peset, V., et al. (2009). Familiarity-based recognition in the young, healthy elderly, mild cognitive impaired and Alzheimer's patients. Neuropsychologia, 47(10), 2056-2064. doi:10.1016/j. neuropsychologia.2009.03.016.

Algarabel, S., Fuentes, M., Escudero, J., Pitarque, A., Peset, V., Mazón, J.-F., et al. (2012). Recognition memory deficits in mild cognitive impairment. Aging, Neuropsychology, and Cognition, 19(5), 608619. doi:10.1080/13825585.2011.640657.

Ally, B. A., Gold, C. A., \& Budson, A. E. (2009). An evaluation of recollection and familiarity in Alzheimer's disease and mild cognitive impairment using receiver operating characteristics. Brain and Cognition, 69(3), 504-513.

American Psychiatric Association (1994). Diagnostic and statistical manual of mental disorders.

Anderson, N. D., Ebert, P. L., Jennings, J. M., Grady, C. L., Cabeza, R., \& Graham, S. J. (2008). Recollection-and familiarity-based memory in healthy aging and amnestic mild cognitive impairment. Neuropsychology, 22(2), 177-187. doi:10.1037/0894-4105.22.2. 177.
Bastin, C., \& Van der Linden, M. (2003). The contribution of recollection and familiarity to recognition memory: a study of the effects of test format and aging. Neuropsychology, 17(1), 14.

Bateman, R. J., Xiong, C., Benzinger, T. L. S., Fagan, A. M., Goate, A., Fox, N. C., et al. (2012). Clinical and biomarker changes in dominantly inherited Alzheimer's disease. New England Journal of Medicine, 367(9), 795-804. doi:10.1056/NEJMoa1202753.

Belleville, S., Ménard, M.-C., \& Lepage, E. (2011). Impact of novelty and type of material on recognition in healthy older adults and persons with mild cognitive impairment. Neuropsychologia, 49(10), 28562865.

Bowles, B., Crupi, C., Mirsattari, S. M., Pigott, S. E., Parrent, A. G., Pruessner, J. C., et al. (2007). Impaired familiarity with preserved recollection after anterior temporal-lobe resection that spares the hippocampus. Proceedings of the National Academy of Sciences, 104(41), 16382-16387. doi:10.1073/pnas.0705273104.

Bowles, B., Crupi, C., Pigott, S., Parrent, A., Wiebe, S., Janzen, L., et al. (2010). Double dissociation of selective recollection and familiarity impairments following two different surgical treatments for temporal-lobe epilepsy. Neuropsychologia, 48(9), $2640-2647$.

Bowles, B., O’Neil, E. B., Mirsattari, S. M., Poppenk, J., \& Köhler, S. (2011). Preserved hippocampal novelty responses following anterior temporal-lobe resection that impairs familiarity but spares recollection. Hippocampus, 21(8), 847-854. doi:10.1002/hipo.20800.

Braak, H., \& Braak, E. (1991). Neuropathological stageing of Alzheimerrelated changes. Acta Neuropathologica, 82(4), 239-259. doi:10. 1007/bf00308809.

Budson, A. E., Daffner, K. R., Desikan, R., \& Schacter, D. L. (2000). When false recognition is unopposed by true recognition: gist-based memory distortion in Alzheimer's disease. Neuropsychology, 14(2), 277.

Chételat, G., La Joie, R., Villain, N., Perrotin, A., de La Sayette, V., Eustache, F., et al. (2013). Amyloid imaging in cognitively normal individuals, at-risk populations and preclinical Alzheimer's disease. NeuroImage: Clinical, 2, 356-365.

Collie, A., \& Maruff, P. (2000). The neuropsychology of preclinical Alzheimer's disease and mild cognitive impairment. Neuroscience \& Biobehavioral Reviews, 24(3), 365-374.

Craik, F. I., \& McDowd, J. M. (1987). Age differences in recall and recognition. Journal of Experimental Psychology Learning Memory and Cognition, 13(3), 474.

Csernansky, J. G., Hamstra, J., Wang, L., McKeel, D., Price, J. L., Gado, M., et al. (2004). Correlations between antemortem hippocampal volume and postmortem neuropathology in $\mathrm{AD}$ subjects. Alzheimer Disease \& Associated Disorders, 18(4), 190-195.

Curran, T. (2000). Brain potentials of recollection and familiarity. Memory \& Cognition, 28(6), 923-938. doi:10.3758/BF03209340.

Curran, T., \& Cleary, A. M. (2003). Using ERPs to dissociate recollection from familiarity in picture recognition. Cognitive Brain Research, 15(2), 191-205.

Curran, T., DeBuse, C., Woroch, B., \& Hirshman, E. (2006). Combined pharmacological and electrophysiological dissociation of familiarity and recollection. The Journal of Neuroscience, 26(7), 1979-1985. doi:10.1523/jneurosci.5370-05.2006.

Dalla Barba, G. (1997). Recognition memory and recollective experience in Alzheimer's disease. Memory, 5(6), 657-672. doi:10.1080/ 741941546

Daselaar, S. M., Fleck, M. S., \& Cabeza, R. (2006a). Triple dissociation in the medial temporal lobes: recollection, familiarity, and novelty. Journal of Neurophysiology, 96(4), 1902-1911. doi:10.1152/jn. 01029.2005

Daselaar, S. M., Fleck, M. S., Dobbins, I. G., Madden, D. J., \& Cabeza, R. (2006b). Effects of healthy aging on hippocampal and rhinal memory functions: an event-related fMRI study. Cerebral Cortex, 16(12), 1771-1782. 
Davidson, P. R., \& Glisky, E. (2002). Neuropsychological correlates of recollection and familiarity in normal aging. Cognitive, Affective, \& Behavioral Neuroscience, 2(2), 174-186. doi:10.3758/CABN.2.2.174.

Dede, A.J., Squire, L.R., \& Wixted, J.T. (2013). A novel approach to an old problem: Analysis of systematic errors in two models of recognition memory. Neuropsychologia

Diana, R. A., Yonelinas, A. P., \& Ranganath, C. (2007). Imaging recollection and familiarity in the medial temporal lobe: a threecomponent model. Trends in Cognitive Sciences, 11(9), 379-386. doi:10.1016/j.tics.2007.08.001.

Donaldson, W. (1996). The role of decision processes in remembering and knowing. Memory \& Cognition, 24(4), 523-533. doi:10.3758/ BF03200940.

Driscoll, I., Hamilton, D. A., Petropoulos, H., Yeo, R. A., Brooks, W. M., Baumgartner, R. N., et al. (2003). The aging hippocampus: cognitive, biochemical and structural findings. Cerebral Cortex, 13(12), 1344-1351.

Duarte, A., Ranganath, C., \& Knight, R. T. (2005). Effects of unilateral prefrontal lesions on familiarity, recollection, and source memory. The Journal of Neuroscience, 25(36), 8333-8337. doi:10.1523/ jneurosci.1392-05.2005.

Dunn, J. C. (2004). Remember-know: a matter of confidence. Psychological Review, 111(2), 524-542. doi:10.1037/0033-295X. 111.2.524.

Easton, A., Zinkivskay, A., \& Eacott, M. J. (2009). Recollection is impaired, but familiarity remains intact in rats with lesions of the fornix. Hippocampus, 19(9), 837-843. doi:10.1002/hipo.20567.

Eldridge, L. L., Knowlton, B. J., Furmanski, C. S., Bookheimer, S. Y., \& Engel, S. A. (2000). Remembering episodes: a selective role for the hippocampus during retrieval. Nature Neuroscience, 3(11), 11491152.

Embree, L. M., Budson, A. E., \& Ally, B. A. (2012). Memorial familiarity remains intact for pictures but not for words in patients with amnestic mild cognitive impairment. Neuropsychologia, 50(9), 2333-2340.

Fabiani, M., \& Friedman, D. (1996). Dissociations between memory for temporal order and recognition memory in aging. Neuropsychologia, 35(2), 129-141.

Fischer, P., Jungwirth, S., Zehetmayer, S., Weissgram, S., Hoenigschnabl, S., Gelpi, E., et al. (2007). Conversion from subtypes of mild cognitive impairment to Alzheimer dementia. Neurology, 68(4), 288-291. doi:10.1212/01.wnl.0000252358.03285.9d.

Folstein, M. F., Folstein, S. E., \& McHugh, P. R. (1975). Mini-Mental State: a practical method for grading the cognitive state of patients for the clinician. Oxford: Pergamon Press.

Fortin, N. J., Wright, S. P., \& Eichenbaum, H. (2004). Recollection-like memory retrieval in rats is dependent on the hippocampus. Nature, 431(7005), 188-191.

Gardiner, J. M. (1988). Functional aspects of recollective experience. Memory \& Cognition, 16(4), 309-313. doi:10.3758/BF03197041.

Gauthier, S., Reisberg, B., Zaudig, M., Petersen, R. C., Ritchie, K., Broich, K., et al. (2006). Mild cognitive impairment. The Lancet, 367(9518), 1262-1270. doi:10.1016/s0140-6736(06)68542-5.

Gomez-Isla, T., Price, J. L., McKeel, D. W., Jr., Morris, J. C., Growdon, J. H., \& Hyman, B. T. (1996). Profound loss of layer II entorhinal cortex neurons occurs in very mild alzheimer, Äôs disease. The Journal of Neuroscience, 16(14), 4491-4500.

Grady, C., McIntosh, A., Horwitz, B., Maisog, J., Ungerleider, L., Mentis, M., et al. (1995). Age-related reductions in human recognition memory due to impaired encoding. Science, 269(5221), 218-221. doi: $10.1126 /$ science. 7618082 .

Greenaway, M. C., Lacritz, L. H., Binegar, D., Weiner, M. F., Lipton, A., \& Cullum, C. M. (2006). Patterns of verbal memory performance in mild cognitive impairment, Alzheimer disease, and normal aging. Cognitive and Behavioral Neurology, 19(2), 79-84.
Grober, E., Buschke, H., Crystal, H., Bang, S., \& Dresner, R. (1988). Screening for dementia by memory testing. Neurology, 38(6), 900 900.

Hansson, O., Zetterberg, H., Buchhave, P., Londos, E., Blennow, K., \& Minthon, L. (2006). Association between CSF biomarkers and incipient Alzheimer's disease in patients with mild cognitive impairment: a follow-up study. The Lancet Neurology, 5(3), 228-234.

Hedges, L. V., Olkin, I., Statistiker, M., Olkin, I., \& Olkin, I. (1985). Statistical methods for meta-analysis. New York: Academic Press New York.

Henson, R. N. A., Rugg, M. D., Shallice, T., Josephs, O., \& Dolan, R. J. (1999). Recollection and familiarity in recognition memory: an event-related functional magnetic resonance imaging study. The Journal of Neuroscience, 19(10), 3962-3972.

Hudon, C., Belleville, S., \& Gauthier, S. (2009). The assessment of recognition memory using the Remember/Know procedure in amnestic mild cognitive impairment and probable Alzheimer's disease. Brain and Cognition, 70(1), 171-179.

Huron, C., Danion, J.-M., Giacomoni, F., Grange, D., et al. (1995). Impairment of recognition memory with, but not without, conscious recollection in schizophrenia. The American Journal of Psychiatry, 152(12), 1737-1742.

Jack, C. R., Knopman, D. S., Jagust, W. J., Shaw, L. M., Aisen, P. S., Weiner, M. W., et al. (2010). Hypothetical model of dynamic biomarkers of the Alzheimer's pathological cascade. The Lancet Neurology, 9(1), 119-128. doi:10.1016/s1474-4422(09)70299-6.

Jacoby, L. L. (1991). A process dissociation framework: separating automatic from intentional uses of memory. Journal of Memory and Language, 30(5), 513-541.

Jacoby, L. L., Toth, J. P., \& Yonelinas, A. P. (1993). Separating conscious and unconscious influences of memory: measuring recollection. Journal of Experimental Psychology: General, 122(2), 139.

Jennings, J. M., \& Jacoby, L. L. (1997). An opposition procedure for detecting age-related deficits in recollection: telling effects of repetition. Psychology and Aging, 12(2), 352.

King, J. A., Trinkler, I., Hartley, T., Vargha-Khadem, F., \& Burgess, N. (2004). The Hippocampal role in spatial memory and the familiarityrecollection distinction: a case study. Neuropsychology, 18(3), 405417. doi:10.1037/0894-4105.18.3.405.

Landau, S. M., Mintun, M. A., Joshi, A. D., Koeppe, R. A., Petersen, R. C., Aisen, P. S., et al. (2012). Amyloid deposition, hypometabolism, and longitudinal cognitive decline. Annals of Neurology, 72(4), $578-586$.

Larrieu, S., Letenneur, L., Orgogozo, J. M., Fabrigoule, C., Amieva, H., Le Carret, N., et al. (2002). Incidence and outcome of mild cognitive impairment in a population-based prospective cohort. Neurology, 59(10), 1594-1599. doi:10.1212/01.wnl.0000034176.07159.f8.

Lopez, O. L., Becker, J. T., Klunk, W., Saxton, J., Hamilton, R. L., Kaufer, D. I., et al. (2000). Research evaluation and diagnosis of probable Alzheimer's disease over the last two decades: I. Neurology, 55(12), 1854-1862. doi:10.1212/wnl.55.12.1854.

Mandler, G. (1980). Recognizing: The judgment of previous occurrence. American Psychological Association

Martin, C. B., Bowles, B., Mirsattari, S. M., \& Köhler, S. (2011). Selective familiarity deficits after left anterior temporal-lobe removal with hippocampal sparing are material specific. Neuropsychologia, 49(7), 1870-1878.

McCabe, D. P., Roediger, H. L., III, McDaniel, M. A., \& Balota, D. A. (2009). Aging reduces veridical remembering but increases false remembering: neuropsychological test correlates of rememberknow judgments. Neuropsychologia, 47(11), 2164-2173.

McElree, B., Dolan, P. O., \& Jacoby, L. L. (1999). Isolating the contributions of familiarity and source information to item recognition: a time course analysis. Journal of Experimental Psychology Learning Memory and Cognition, 25(3), 563-582. doi:10.1037/0278-7393. 25.3.563. 
McKhann, G., Drachman, D., Folstein, M., Katzman, R., Price, D., \& Stadlan, E. M. (1984). Clinical diagnosis of Alzheimer's disease: report of the NINCDS-ADRDA work group* under the auspices of department of health and human services task force on Alzheimer's disease. Neurology, 34(7), 939. doi:10.1212/wnl.34.7.939.

Mikhailova, E. S., Vladimirova, T. V., Iznak, A. F., Tsusulkovskaya, E. J., \& Sushko, N. V. (1996). Abnormal recognition of facial expression of emotions in depressed patients with major depression disorder and schizotypal personality disorder. Biological Psychiatry, 40(8), 697-705.

Mitchell, A. J. (2009). A meta-analysis of the accuracy of the mini-mental state examination in the detection of dementia and mild cognitive impairment. Journal of Psychiatric Research, 43(4), 411-431.

Morris, J. C. (2006). Mild cognitive impairment is early-stage Alzheimer disease: time to revise diagnostic criteria. Archives of Neurology, 63(1), 15-16. doi:10.1001/archneur.63.1.15.

Morris, J. C., Storandt, M., Miller, J. P., McKeel, D. W., Price, J. L., Rubin, E. H., et al. (2001). Mild cognitive impairment represents early-stage Alzheimer disease. Archives of Neurology, 58(3), 397405. doi:10.1001/archneur.58.3.397.

Nordlund, A., Rolstad, S., Klang, O., Edman, Å., Hansen, S., \& Wallin, A. (2010). Two-year outcome of MCI subtypes and aetiologies in the Göteborg MCI study. Journal of Neurology, Neurosurgery \& Psychiatry, 81(5), 541-546.

Petersen, R. C. (2004). Mild cognitive impairment as a diagnostic entity. Journal of Internal Medicine, 256(3), 183-194. doi:10.1111/j.13652796.2004.01388.x.

Petersen, R. C., Aisen, P. S., Beckett, L. A., Donohue, M. C., Gamst, A. C., Harvey, D. J., et al. (2010). Alzheimer's disease neuroimaging initiative (ADNI) clinical characterization. Neurology, 74(3), 201209.

Petersen, R. C., Doody, R., Kurz, A., Mohs, R. C., Morris, J. C., Rabins, P. V., et al. (2001a). Current concepts in mild cognitive impairment. Archives of Neurology, 58(12), 1985-1992. doi:10.1001/archneur. 58.12.1985.

Petersen, R. C., Doody, R., Kurz, A., Mohs, R. C., Morris, J. C., Rabins, P. V., et al. (2001b). Current concepts in mild cognitive impairment. Archives of Neurology, 58(12), 1985-1992. doi:10.1001/archneur. 58.12.1985.

Petersen, R. C., \& Morris, J. C. (2005). Mild cognitive impairment as a clinical entity and treatment target. Archives of Neurology, 62(7), 1160-1163. doi:10.1001/archneur.62.7.1160.

Petersen, R. C., Smith, G. E., Waring, S. C., Ivnik, R. J., Tangalos, E. G., \& Kokmen, E. (1999). Mild cognitive impairment: clinical characterization and outcome. Archives of Neurology, 56(3), 303.

Prull, M. W., Dawes, L. L. C., Martin Iii, A. M., Rosenberg, H. F., \& Light, L. L. (2006). Recollection and familiarity in recognition memory: adult age differences and neuropsychological test correlates. Psychology and Aging, 21(1), 107-118. doi:10.1037/08827974.21.1.107.

Rabinowitz, J. C. (1984). Aging and recognition failure. Journal of Gerontology, 39(1), 65-71. doi:10.1093/geronj/39.1.65.

Ranganath, C., Yonelinas, A. P., Cohen, M. X., Dy, C. J., Tom, S. M., \& D'Esposito, M. (2004). Dissociable correlates of recollection and familiarity within the medial temporal lobes. Neuropsychologia, 42(1), 2-13.

Rauchs, G., Piolino, P., Mézenge, F., Landeau, B., Lalevée, C., Pélerin, A., et al. (2007). Autonoetic consciousness in Alzheimer's disease: neuropsychological and PET findings using an episodic learning and recognition task. Neurobiology of Aging, 28(9), 1410-1420

Ravaglia, G., Forti, P., Maioli, F., Martelli, M., Servadei, L., Brunetti, N., et al. (2006). Conversion of mild cognitive impairment to dementia: predictive role of mild cognitive impairment subtypes and vascular risk factors. Dementia and Geriatric Cognitive Disorders, 21(1), $51-58$.
Raz, N., Lindenberger, U., Rodrigue, K. M., Kennedy, K. M., Head, D., Williamson, A., et al. (2005). Regional brain changes in aging healthy adults: general trends, individual differences and modifiers. Cerebral Cortex, 15(11), 1676-1689.

Rosenthal, R. (1991). Meta-analytic procedures for social research (Vol. 6): Sage.

Sarazin, M., Berr, C., De Rotrou, J., Fabrigoule, C., Pasquier, F., Legrain, S., et al. (2007). Amnestic syndrome of the medial temporal type identifies prodromal AD: a longitudinal study. Neurology, 69(19), 1859-1867. doi:10.1212/01.wnl.0000279336.36610.f7.

Sauvage, M. M., Fortin, N. J., Owens, C. B., Yonelinas, A. P., \& Eichenbaum, H. (2008). Recognition memory: opposite effects of hippocampal damage on recollection and familiarity. Nature Neuroscience, 11(1), 16-18.

Scahill, R. I., Frost, C., Jenkins, R., Whitwell, J. L., Rossor, M. N., \& Fox, N. C. (2003). A longitudinal study of brain volume changes in normal aging using serial registered magnetic resonance imaging. Archives of Neurology, 60(7), 989

Schacter, D. L., Koutstaal, W., \& Norman, K. A. (1997). False memories and aging. Trends in Cognitive Sciences, 1(6), 229-236.

Schonfield, D., \& Robertson, B. A. (1966). Memory storage and aging. Canadian Journal of Psychology/Revue canadienne de psychologie, 20(2), 228.

Seo, S. W., Im, K., Lee, J.-M., Kim, Y.-H., Kim, S. T., Kim, S. Y., et al. (2007). Cortical thickness in single- versus multiple-domain amnestic mild cognitive impairment. NeuroImage, 36(2), 289-297.

Serra, L., Bozzali, M., Cercignani, M., Perri, R., Fadda, L., Caltagirone, C., et al. (2010). Recollection and familiarity in amnesic mild cognitive impairment. Neuropsychology, 24(3), 316-326. doi:10. 1037/a0017654.

Skinner, E. I., \& Fernandes, M. A. (2007). Neural correlates of recollection and familiarity: a review of neuroimaging and patient data. Neuropsychologia, 45(10), 2163-2179.

Slotnick, S. D. (2009). "Remember" source memory ROCs indicate recollection is a continuous process. Memory, 18(1), 27-39. doi: $10.1080 / 09658210903390061$.

Speer, N. K., \& Curran, T. (2007). ERP correlates of familiarity and recollection processes in visual associative recognition. Brain Research, 1174, 97-109.

Squire, L. R., Wixted, J. T., \& Clark, R. E. (2007). Recognition memory and the medial temporal lobe: a new perspective. Nature Reviews Neuroscience, 8(11), 872-883.

Tulving, E. (1985). Memory and consciousness. Canadian Psychology, 26, 1-12.

van der Meulen, M., Lederrey, C., Rieger, S. W., van Assche, M., Schwartz, S., Vuilleumier, P., et al. (2012). Associative and semantic memory deficits in amnestic mild cognitive impairment as revealed by functional magnetic resonance imaging. Cognitive and Behavioral Neurology, 25(4), 195-215.

Vann, S. D., Tsivilis, D., Denby, C. E., Quamme, J. R., Yonelinas, A. P., Aggleton, J. P., et al. (2009). Impaired recollection but spared familiarity in patients with extended hippocampal system damage revealed by 3 convergent methods. Proceedings of the National Academy of Sciences, 106(13), 5442-5447. doi:10.1073/pnas. 0812097106.

Westerberg, C. E., Paller, K. A., Weintraub, S., Mesulam, M. M., Holdstock, J. S., Mayes, A. R., et al. (2006). When memory does not fail: familiarity-based recognition in mild cognitive impairment and Alzheimer's disease. Neuropsychology, 20(2), 193-205. doi:10. 1037/0894-4105.20.2.193.

Whittington, C. J., Podd, J., \& Kan, M. M. (2000). Recognition memory impairment in Parkinson's disease: power and meta-analyses. Neuropsychology, 14(2), 233-246. doi:10.1037/0894-4105.14.2.233.

Winblad, B., Palmer, K., Kivipelto, M., Jelic, V., Fratiglioni, L., Wahlund, L. O., et al. (2004). Mild cognitive impairment - beyond controversies, towards a consensus: report of the international working group 
on mild cognitive impairment. Journal of Internal Medicine, 256(3), 240-246. doi:10.1111/j.1365-2796.2004.01380.x.

Wixted, J. T. (2007). Dual-process theory and signal-detection theory of recognition memory. Psychological Review, 114(1), 152.

Wixted, J. T., \& Mickes, L. (2010). A continuous dual-process model of remember/know judgments. Psychological Review, 117(4), 1025.

Wolf, H., Grunwald, M., Ecke, G. M., Zedlick, D., Bettin, S., Dannenberg, C., et al. (1998). The prognosis of mild cognitive impairment in the elderly. J Neural Transm Supp, 54, $31-50$.

Wolk, D. A., Dunfee, K. L., Dickerson, B. C., Aizenstein, H. J., \& DeKosky, S. T. (2011). A medial temporal lobe division of labor: insights from memory in aging and early Alzheimer disease. Hippocampus, 21(5), 461-466. doi:10.1002/hipo.20779.

Wolk, D. A., Mancuso, L., Kliot, D., Arnold, S. E., \& Dickerson, B. C. (2013). Familiarity-based memory as an early cognitive marker of preclinical and prodromal AD. Neuropsychologia, 51(6), 1094 1102.

Wolk, D. A., Signoff, E. D., \& DeKosky, S. T. (2008). Recollection and familiarity in amnestic mild cognitive impairment: a global decline in recognition memory. Neuropsychologia, 46(7), 1965-1978.

Woodruff, C. C., Hayama, H. R., \& Rugg, M. D. (2006). Electrophysiological dissociation of the neural correlates of recollection and familiarity. Brain Research, 1100(1), 125135.

Yonelinas, A. P. (2002). The Nature of Recollection and Familiarity: A Review of 30 Years of Research. Journal of Memory and Language, 46(3), 441-517. doi:10.1006/jmla.2002.2864.
Yonelinas, A. P., Dobbins, I., Szymanski, M. D., Dhaliwal, H. S., \& King, L. (1996). Signal-detection, threshold, and dual-process models of recognition memory: ROCs and conscious recollection. Consciousness and Cognition, 5(4), 418-441.

Yonelinas, A. P., \& Jacoby, L. L. (1994). Dissociations of processes in recognition memory: effects of interference and of response speed. Canadian journal of experimental psychology $=$ Revue canadienne de psychologie experimentale, 48(4), 516-535.

Yonelinas, A. P., \& Jacoby, L. L. (1995). The relation between remembering and knowing as bases for recognition: effects of size congruency. Journal of Memory and Language, 34(5), 622-643.

Yonelinas, A. P., Kroll, N. E. A., Dobbins, I., Lazzara, M., \& Knight, R. T. (1998). Recollection and familiarity deficits in amnesia: convergence of remember-know, process dissociation, and receiver operating characteristic data. Neuropsychology, 12(3), 323-339. doi:10. 1037/0894-4105.12.3.323.

Yonelinas, A. P., Kroll, N. E. A., Quamme, J. R., Lazzara, M. M., Sauve, M. J., Widaman, K. F., et al. (2002). Effects of extensive temporal lobe damage or mild hypoxia on recollection and familiarity. Nature Neuroscience, 5(11), 1236-1241.

Yonelinas, A. P., Otten, L. J., Shaw, K. N., \& Rugg, M. D. (2005). Separating the brain regions involved in recollection and familiarity in recognition memory. The Journal of Neuroscience, 25(11), 3002 3008. doi:10.1523/jneurosci.5295-04.2005.

Yonelinas, A. P., Widaman, K. F., Mungas, D., Reed, B., Weiner, M. W., \& Chui, H. C. (2007). Memory in the aging brain: doubly dissociating the contribution of the hippocampus and entorhinal cortex. Hippocampus, 17(11), 1134-1140. doi:10.1002/hipo.20341. 